\title{
Oscillations and uniaxial mechanochemical waves in a model of an active poroelastic medium: Application to deformation patterns in protoplasmic droplets of Physarum polycephalum
}

\author{
Sergio Alonso ${ }^{\mathrm{a}, \mathrm{b}}$, Ulrike Strachauer ${ }^{\mathrm{c}, \mathrm{d}}$, Markus Radszuweit ${ }^{\mathrm{b}, \mathrm{e}}$, Markus Bär ${ }^{\mathrm{b}}$, \\ Marcus J. B. Hauser ${ }^{\mathrm{C}}$ \\ ${ }^{a}$ Department of Applied Physics, Universitat Politécnica de Catalunya, Av. Dr. Marañón \\ 44, E-08028 Barcelona, Spain. \\ ${ }^{b}$ Physikalisch-Technische Bundesanstalt, Abbestrasse 2-12, 10587 Berlin, Germany. \\ ${ }^{c}$ Biophysics Group, Institute of Experimental Physics, Otto-von-Guericke-Universität \\ Magdeburg, Universitätsplatz 2, 39106, Magdeburg, Germany. \\ ${ }^{d}$ Department of Aquatic Ecosystem Analysis and Management (ASAM), Helmholtz Centre \\ for Environmental Research (UFZ), Brückstrasse 3a, 39114 Magdeburg, Germany \\ ${ }^{e}$ Weierstrass Institute for Applied Analysis and Stochastics, Mohrenstrasse 39, 10117 \\ Berlin, Germany.
}

\begin{abstract}
Self-organization in cells often manifests itself in oscillations and waves. Here, we address deformation waves in protoplasmic droplets of the plasmodial slime mold Physarum polycephalum by modelling and experiments. In particular, we extend a one-dimensional model that considered the cell as an poroelastic medium, where active tension caused mechanochemical waves that were regulated by an inhibitor (M. Radszuweit et al., Phys. Rev. Lett. 110 (2013), 138102). Our extension consists of a simple, qualitative chemical reaction-diffusion model (Brusselator) that describes the regulation of the inhibitor by another biochemical species. The biochemical reaction enhances the formation of mechanochemical waves if the reaction rates and input concentrations are near or inside an oscillatory regime. The period of the waves is found to be controlled by the characteristic oscillation period, whereas their wavelength is set by mechanical parameters. The model also allows for a systematic study of the chemical
\end{abstract}

\footnotetext{
* Corresponding author

Email addresses: s.alonso@upc.edu (Sergio Alonso), Markus.Baer@ptb.de (Markus Bär), marcus.hauser@ovgu.de (Marcus J. B. Hauser) 
activity at the onset of mechanochemical waves. We also present examples for pattern formation in protoplasmic droplets of Physarum polycephalum including global oscillations where the central region of the droplets is in antiphase to the boundary zone, as well as travelling and standing wave like uniaxial patterns. Finally, we apply our model to reproduce these experimental results by identifying the active tension inhibitor with the intracellular calcium concentration in the Physarum droplets and by using parameter values from mechanical experiments, respectively knowledge about the properties of calcium oscillations in Physarum. The simulation results are then found to be in good agreement with the experimental observations.

Keywords: Pattern formation, Active gels, Physarum polycephalum, Biological cells, Cell motility, Poroelastic medium

\section{Introduction}

Biological cells are dynamic objects, the internal organization of which is highly structured. The intracellular environment is compartmented, and a very large number of biochemical substances react in the various compartments, diffuse through a crowded environment, and move following gradients in chemical potentials or due to mechanical and hydrodynamic forces. In such a complex system, the spatial distribution of the biochemical substances becomes a key factor for the correct performance of the cellular activities.

Biochemical self-organization manifests itself in the formation of static and dynamic structures. Pattern-forming processes are typically associated with the interplay between biochemical reactions and the transport of molecules, which may be either passive or active. When a molecule (or particle) is transported due to intracellular concentration gradients, the transport is passive, e.g., during thermal diffusion of molecules. On the other hand, particles can also be transported actively, i.e., when the cell consumes energy to transport the particle. This is the case when molecules (or vesicles, etc.) are transported as a result of the action of active mechanical forces produced by molecular motors 
[1]. These molecular machines pull at the filaments which form the cytoskeleton of the cell. A combination of such forces and diffusion of biochemical regulators may induce the formation of static and dynamic patterns in active fluid [2] and poroelastic cellular media [3]. Similarly, oscillatory instabilities were found for models of active gels [4] as well as for models of mechanical deformations in epithelial tissues $[5,6,7]$.

The filaments of the cytoskeleton consist of chains of proteins like actin and are kept together in bundles by cross-linking proteins [8]. At large time scales, the cytoskeleton is dynamic and fluidized. There is a continuous polymerization and depolymerization of filaments and the unbinding of cross-linkers may produce cytoplasmic flows. Models based on active gel theory successfully describe such flows $[9,10]$.

At smaller time scales, however, the cytoskeleton is more rigid and behaves as an elastic solid. Together, the cytoskeleton and the cytosol, i.e., the fluid part of the cytoplasm, form a poroelastic material. The dominance of the poroelasticity at small time scales has been shown in experiments on partial dehydration of living cells [11] and by the study of the cell response to small perturbations measured by atomic force microscopy [12].

Physarum polycephalum has been used as a model organism to analyze the filament structure of the cytoskeleton $[13,14,15]$. Physarum is a slime mold (myxomycetes) that forms giant multinucleate amoeboid cells that may reach vastly differing sizes (from a few $\mu m^{2}$ to a $m^{2}$ ). Different aspects of the selforganization of this organism may be investigated by varying the cell size. For instance, extended plasmodia form complex tubular transportation networks to feed protoplasm to and from remote parts of the organism. The morphology of such networks is analyzed from the point of view of the physiology $[14,16$, $17,18]$ as well as in the frame of graph theory $[19,20,21,22,23]$. On the other hand, the scientific focus also lies on small cellular structures of about 50 - $800 \mu m$ length, where the amoeboid motility of migrating microplasmodia $[24,25,26,27,28,29,30]$ is currently intensively investigated.

In addition to extended networks and the motility of migrating microplas- 
modia, small protoplasmic droplets of Physarum have been studied in a third line of research. Generally, aliquots of $0.5-25 \mu l$ protoplasm are extracted either from a strand (vein) of an extended network [31] or from its apical zone [32] and transferred into a Petri dish, where they expand into a roughly circular area to generate a protoplasmic droplet of $400 \mu m-1 \mathrm{~mm}$ diameter. Such droplets are subjected to reorganization and intracellular differentiation and will eventually form a new, viable cell. Immediately after deposition of the cell material on the Petri dish, it forms a plasma membrane and then begins to self-organize. During this process, mechanical deformation waves travel through the droplet $[31,32,33]$ and actively take part in the cellular reorganization. The loci and instants of the onset of cell dilation (i.e., of the increase in cell thickness) are tightly correlated to peaks of intracellular calcium concentration [32].

In Physarum, the driving force for pumping protoplasm through the cell is provided by coherent contractions of the cytoskelton. The dynamics of these motor proteins is regulated by the intracellular concentration of calcium [32, 34, 35 ], because an elevation in the calcium concentration leads to a delation of the cell due to the relaxation of the actin fibers [35, 36]. Whereas the regulation exerted by calcium on the cytoskeleton dynamics is well established [35, 36, $37,38]$, less is known about the mechanistic details connecting the calcium oscillations with the cytoskeletal dynamics. This is reflected by the current discussion if the cytoskeleton dynamics is "merely" coupled to an autonomous, primary calcium oscillator [39, 40, 41] (for instance, oscillations of mitochondrial activity [40,41], which also cause the concentration of cytosolic calcium to oscillate) or if the contractility and the resulting protoplasmic flows need to be considered as a part of the feedback loop leading to calcium oscillations $[35,42,43]$.

Very recently, models based on the poroelastic nature of Physarum cell have been proposed to rationalize the mechanism of locomotion of migrating microplasmodia [27, 28]. The concept of Physarum cells as being poroelastic media was used previously in a series of studies aiming at understanding the dynamics of the reorganization of protoplasmic droplets and the related deformation 
patterns $[3,44,45]$.

The combination of the poroelastic properties [12] with the concept of active matter has recently been applied for eukaryotic cells predicting mechanochemical waves [3]. In the present paper the model for intracellular mechanochemical waves will be used as a starting point and extended by an oscillatory chemical reaction to describe deformation patterns in protoplasmic droplets of Physarum polycephalum. To achieve this, we describe a generic model of an active poroelastic medium regulated by a diffusive biochemical substance that inhibits mechanical tension. This regulator represents the intracellular concentration of free calcium ions in Physarum droplets. This model is then extended by the incorporation of a biochemical oscillator in the dynamics of the regulator to allow for calcium oscillations that are believed to be essential for deformation patterns in Physarum (see [45] and references therein).

The present work focuses on uniaxial patterns and is therefore constraint to a single spatial dimension and is complimentary to an earlier study [45], that coupled a realistic calcium oscillator to a two-dimensional version of the active poroelastic model. Since it is controversial if Physarum droplets contain a genuine oscillator, one main aim of our study here is to compare the behavior of systems with oscillatory and non-oscillatory calcium regulation. In principle, mechanical waves can appear also for conserved total concentration of free calcium ions. Such an absence of the local calcium concentration is, however, unrealistic for living cells. First, we study the resulting model for typical eukaryotic cell parameters and discuss its general properties. Next, we show some experimental results on mechano-chemical waves in protoplasmic droplets of Physarum polycephalum. And finally, we employ the model of the poroelastic medium coupled to a calcium oscillator to reproduce the particular experimental patterns observed in protoplasmic droplets of Physarum by using a parameter set typical for the intracellular dynamics of Physarum. We find that the typical wavelengths of the mechanochemical deformation waves are determined by the viscoelastic properties of the model, whereas the period of the oscillations is controlled by the frequency of the driving calcium oscilla- 
tor. For large mechanochemical feedback strengths, waves appear already for non-oscillatory calcium dynamics. Oscillatory conditions, however, lower the threshold for the occurrence of deformation patterns substantially. Altogether, the model studied demonstrates that deformation waves are more likely to occur in a situation where chemical oscillations are present in an active poroelastic medium.

\section{Active poroelastic model with chemical reactions}

\subsection{Mechanical stresses}

Following earlier work [3], we assume that the interior of the cells, i.e., the cytoplasm, is mainly formed by two phases, namely the cytoskeleton corresponding to the solid or gel phase - and the cytosol representing the fluid or sol phase. For clarity of terminology, we will use the terms gel for the cytoskeleton and fluid for the cytosol and the corresponding labels $g$ and $f$ throughout. Therefore, the total stress $\sigma$ inside the cytoplasm is the sum of the stress in the cytoskeletal phase $\sigma_{g}$ and the stress in the cytosol $\sigma_{f}$ :

$$
\sigma=\rho_{g} \sigma_{g}+\rho_{f} \sigma_{f}
$$

where the the respective fractions of cytosol (fluid) and cytoskeleton (gel), $\rho_{f}$ and $\rho_{g}$, are assumed to be constant and shall satisfy $\rho_{f}+\rho_{g}=1$. Both phases have different structural and mechanical properties:

- The cytoskeleton is a viscoelastic active gel formed by a network of interconnected actin filaments [8]. The filaments are interconnected by crosslinkers and molecular motors, which produce an active tension $(T)$ in the network. To be active, the molecular motors require ATP, which we consider here to be abundant and to be of constant concentration. The molecular motors are regulated by several molecules. The location of such regulators play an important role in the control of the tension in the cytoskeleton. The active tension produced by the motors is inhibited by one 
regulator such that $T=T_{0}-\xi f(c)$, where $c$ corresponds to the concentration of the regulator, $T_{0}$ is the tension at low $c$, and $\xi$ is the coupling strength between the concentration $c$ and the active tension $T$. In this paper, we will assume that $T$ depends only on the regulating species; for a recent model introducing two antagonistic regulators in an active fluid see [51].

The active gel has viscoelastic properties. We assume that the cytoskeleton is a viscoelastic solid gel [46] in contrast to other studies which consider the cytoskeleton a viscoelastic fluid [47]. Our approach call for the use of the Kelvin-Voigt model for the description of the viscoelasticity of the active gel. The viscoelastic stress of the gel phase together with the active tension $T$ produced by the myosin motors reads:

$$
\sigma_{g}=E \epsilon+\eta_{g} \dot{\epsilon}+T
$$

where $\epsilon$ is the strain, $E$ is the Young modulus, and $\eta_{g}$ is the viscous dampening coefficient of the gel. Using linear elasticity theory [48], assuming that $|\epsilon| \ll 1$, as is the case for small strains, we relate the strain $\epsilon=\partial_{x} u$ with the local displacement field $u$. This field indicates the deformation of the actin network. The stress is rewritten in terms of the displacement field:

$$
\sigma_{g}=E \partial_{x} u+\eta_{g} \partial_{x} \dot{u}+T
$$

- The cytosol is the viscous fluid part of the cytoplasm. We assume that the gel is a poroelastic solid material [49], penetrated by the cytosol. The stress in the fluid phase is just viscous:

$$
\sigma_{f}=\eta_{f} \partial_{x} v ;
$$

where $v$ is the velocity field of the fluid and $\eta_{f}$ the viscosity of the fluid phase. The regulators of the active tension of the cytoskeleton are transported by both advection and diffusion through the cytosol. 


\subsection{Poroelastic model}

The equations of the force balance of both phases are:

$$
\begin{aligned}
& \rho_{g} \partial_{x} \sigma_{g}=f_{g}+\rho_{g} \partial_{x} p, \\
& \rho_{f} \partial_{x} \sigma_{f}=f_{f}+\rho_{f} \partial_{x} p,
\end{aligned}
$$

where $f_{f}$ and $f_{g}$ are the drag forces of the cytosol and the cytoskeleton, respectively, and $p$ is the pressure that is formally introduced by imposing the incompressibility condition of the whole poroelastic system [49]. We have assumed Darcy's law for a porous medium to derive the drag force:

$$
f_{f}=\rho_{f} \rho_{g} \beta(v-\dot{u}),
$$

where the parameter $\beta$ is the ratio between the viscosity of the fluid $\left(\eta_{f}\right)$ and its permeability $(\kappa)$. The permeability corresponds basically to the square of the cytoskeleton mesh size $\ell_{m}$. Due to the symmetry of the multiphase system we obtain $f_{g}=-f_{f}$. Inserting Eq. (3) and (4) into Eq. (5), we obtain:

$$
\begin{aligned}
\rho_{g}\left(E \partial_{x}^{2} u+\eta_{g} \partial_{x}^{2} \dot{u}+\partial_{x} T\right) & =\rho_{f} \rho_{g} \beta(\dot{u}-v)+\rho_{g} \partial_{x} p, \\
\rho_{f} \eta_{f} \partial_{x}^{2} v & =\rho_{f} \rho_{g} \beta(v-\dot{u})+\rho_{f} \partial_{x} p ;
\end{aligned}
$$

The pressure in Eqs. (7) can be eliminated by subtracting the first from the second equation, and finally the balance equation reads:

$$
\eta_{f} \partial_{x}^{2} v-\eta_{g} \partial_{x}^{2} \dot{u}-E \partial_{x}^{2} u-\partial_{x} T-\beta(v-\dot{u})=0 ;
$$

This relation couples the dynamics of both phases. To close the system of equations we employ the incompressibility condition $[3,49]$ of the fluid-gel system:

$$
\partial_{x}\left(\rho_{f} v+\rho_{g} \dot{u}\right)=0 .
$$

Thus, the dynamics of the poroelastic medium can be obtained from the analysis of Eqs. (8-9).

However, for some boundary conditions the incompressibility condition reduces to a simpler form. Assuming zero fluid velocity $v=0$ and zero displacement field $u=0$ at the boundaries, Eq.(9) yields a relation between the shift 
field and the fluid velocity:

$$
\rho_{g} \dot{u}=-v \rho_{f} .
$$

Eq. (10) is the core of the poroelastic approximation: the flux of fluid in one direction produces a corresponding flux of gel in the opposite direction.

\subsection{Body reference system}

We define the body reference velocity $w=v-\dot{u}$. In this new reference frame, Eq. (8) reads

$$
\eta \partial_{x}^{2} w-E \partial_{x}^{2} u-\partial_{x} T-\beta w=0
$$

where the viscosity is renormalized according to $\eta=\rho_{f} \eta_{g}+\rho_{g} \eta_{f}$. In this reference frame both velocities can be explicitly expressed in terms of the body reference velocity:

$$
\partial_{t} u=-w \rho_{f}, \quad v=w \rho_{g} .
$$

\subsection{Biochemical oscillator}

The fluid (cytosolic) phase contains small molecules which may perform regulatory function. A regulator species diffuses and is advected by the fluid phase, such that its concentration $c$ obeys:

$$
\partial_{t} c+\partial_{x}(w c)=D_{c} \partial_{x}^{2} c,
$$

where $D_{c}$ is the diffusion coefficient of the regulator species. In the present paper, we consider that either calcium ions or any other species whose concentrations are governed or regulated by them act as the "regulator species" c. Although other ions like magnesium can also slow down the activity of the molecular motor myosin V [50], we will use the term "regulator species" $c$ and calcium as synonyms.

The regulation of the activity of the cell can be performed by increasing or decreasing the active tension $T$. We consider that both the activity of the molecular motors and the active tension $T$ are decreased as the concentration 
of the regulator species $c$ (calcium) increases. We model this effect by the expression:

$$
T=T_{o}-\xi \frac{c}{1+c},
$$

where $T_{o}$ is an homogeneous active tension produced by a constant density of molecular motors and $\xi$ is the coupling strength between the concentration $c$ and the active tension. The coupling strength determines if the regulator $(c)$ activates $(\xi<0)$ or inhibits $(\xi>0)$ the active tension. Both cases have been compared in related models of active gels [51].

We consider the dynamics of two biochemical species $c$ and $a$ for calcium and the control species, respectively. On the one hand, calcium ions regulate the active tension of the cell, on the other hand they interact with other components of the medium and produce dynamics that follow non-linear evolution equations. The dynamics of calcium and the control species, whose interplay induces oscillations of the concentration of calcium ions, are modelled by the reaction-advection-diffusion equations $(15,16)$ :

$$
\begin{aligned}
\partial_{t} c+\partial_{x}(w c) & =\psi R_{c}(c, a)+D_{c} \partial_{x}^{2} c \\
\partial_{t} a+\partial_{x}(w a) & =\psi R_{a}(c, a)+D_{a} \partial_{x}^{2} a
\end{aligned}
$$

where $\psi^{-1}$ determines the temporal scale of the dynamics and the functions $R_{c}(c, a)$ and $R_{a}(c, a)$ describe the reaction kinetics of calcium and the control species, respectively. The concentrations $c$ and $a$ can accumulate due to the advection of the fluid phase. An earlier one-dimensional model for mechanochemical waves [3] was based on a constant average concentration of calcium and corresponds to the special case $R_{c}=R_{a}=0$. Such an assumption is, however, not realistic for calcium concentration in living cells, where calcium is always regulated by other species, here summarized in the additional variable $a$. Many previous studies, see e.g. $[39,52]$, show that calcium oscillations are crucial for the intracellular dynamics of Physarum.

The calcium oscillator is accounted for by a model with simple reaction 
terms, following the Brusselator model [53]:

$$
\begin{aligned}
& R_{c}(c, a)=A+c^{2} a-B c-c, \\
& R_{a}(c, a)=-c^{2} a+B c,
\end{aligned}
$$

where the parameters $A$ and $B$ determine the conditions, especially the frequency and amplitude of the oscillations. The homogeneous steady state solution is given by the concentrations $c=c_{o}=A$ and $a=a_{o}=B / A$. This solution is stable for $B<1+A^{2}$ and it becomes unstable through a Hopf bifurcation such that the system oscillates if $B>1+A^{2}$. Here, we keep $A=1$ and we vary the parameter $B$ above and below the critical value $B_{c}=2$. Taking into account the diffusion in the spatially extended Brusselator model, the

homogeneous solution is unstable to a Turing instability if $B>\left(1+A \sqrt{\frac{D_{c}}{D_{a}}}\right)^{2}$, see ref. [54] for more details. The dimensional constant $\psi$ in Eqs. $(15,16)$ is adjusted together with $A$ and $B$ to match the period of the calcium oscillations observed in Physarum. The Turing instability demands that the diffusion coefficient of the control species $a$ is sufficiently larger than that of the regulator $c$, i.e., $D_{a} \gg D_{c}$, which is a condition that is complicated to fulfill, due to the usually large diffusion coefficients of ions associated with the variable $c$. In fact, the the opposite limit $D_{a}<D_{c}$ is the more realistic situation. Therefore, we consider the case where $D_{a}=0$ in the following section whereas the Turing instability is not discussed.

\subsection{Linear stability analysis}

Let us consider the entire set of equations that we have derived so far:

$$
\begin{aligned}
0 & =\eta \partial_{x}^{2} w-E \partial_{x}^{2} u-\partial_{x} T-\beta w, \\
\partial_{t} u & =-w \rho_{f}, \\
\partial_{t} c & =-\partial_{x}(w c)+\psi\left(A+c^{2} a-B c-c\right)+D_{c} \partial_{x}^{2} c, \\
\partial_{t} a & =-\partial_{x}(w a)+\psi\left(-c^{2} a+B c\right),
\end{aligned}
$$

where $\psi$ represents the time scales of the chemical kinetics. The parameter values for Physarum employed below are summarized in Table 1. The spatially 
homogeneous steady state of Eqs. (11-15) is given by constant concentrations $\left(c=c_{o}=A\right.$ and $\left.a=a_{o}=B / A\right)$ in a static two-phase system $(w=0$ and $u=0)$. The linear stability analysis of this steady state with respect to small periodic perturbations $(\delta w, \delta u, \delta c, \delta a) e^{i k x+\lambda t}$ follows from the Jacobian

$$
\left|\begin{array}{cccc}
-\lambda & 0 & 0 & -\rho_{f} \\
0 & \psi(B-1)-D_{c} k^{2}-\lambda & \psi A^{2} & -i k A \\
0 & -\psi B & -\psi A^{2}-\lambda & -i k B / A \\
E k^{2} & \xi i k /(1+A)^{2} & 0 & -\left(\beta+\eta k^{2}\right)
\end{array}\right|=0 .
$$

The resulting three eigenvalues $\lambda_{i}$ determine the evolution of the perturbations and are obtained by solution of the characteristic polynomial:

$$
\begin{array}{r}
\lambda^{3}\left(1+\ell^{2} k^{2}\right) \\
+\lambda^{2}\left[\left(1+\ell^{2} k^{2}\right)\left(\psi A^{2}-\psi B+\psi+D_{c} k^{2}\right)-D_{c} k^{2}\left(F-M \rho_{f}\right)\right] \\
+\lambda\left[\left(1+\ell^{2} k^{2}\right)\left(\psi^{2} A^{2}+D_{c} A^{2} \psi k^{2}\right)-\psi D_{c} k^{2}\left(B+A^{2}\right) F\right. \\
\left.+D_{c} k^{2} M \rho_{f}\left(\psi A^{2}+\psi-\psi B+D_{c} k^{2}\right)\right] \\
+D_{c} k^{2} M \rho_{f}\left(\psi^{2} A^{2}+\psi A^{2} D_{c} k^{2}\right)=0
\end{array}
$$

where we have defined the characteristic length $\ell=\sqrt{\eta / \beta}$, and two nondimensional parameters $M$ and $F$. The non-dimensional elastic parameter

$$
M=\frac{E}{D_{c} \beta},
$$

describes the ratio of the diffusive to elastic time scales and the non-dimensional mechanical parameter

$$
F=\frac{\xi A}{D_{c} \beta(1+A)^{2}},
$$

which is the ratio of the Péclet number to the critical Péclet number for the onset of mechanochemical instability found in the absence of chemical reactions respectively calcium regulation [3]. The quantity $F$ determines the thus relative intensity of the active mechanical force. The Péclet number is $\xi /\left(D_{c} \beta\right)$ and 
(a)

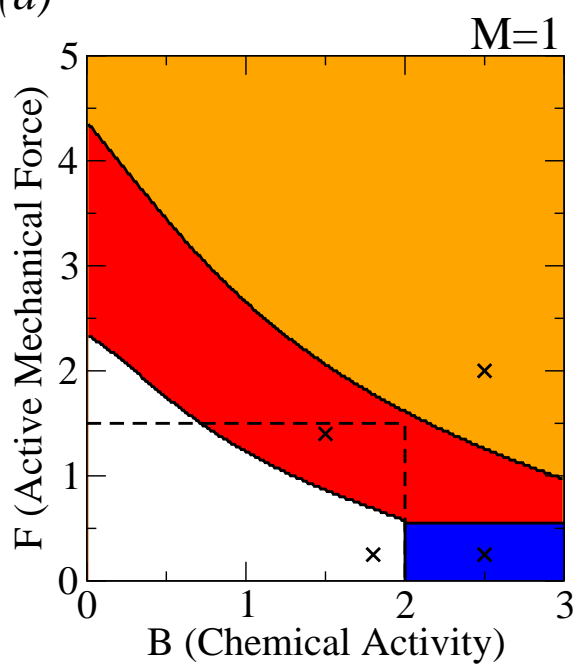

(b)

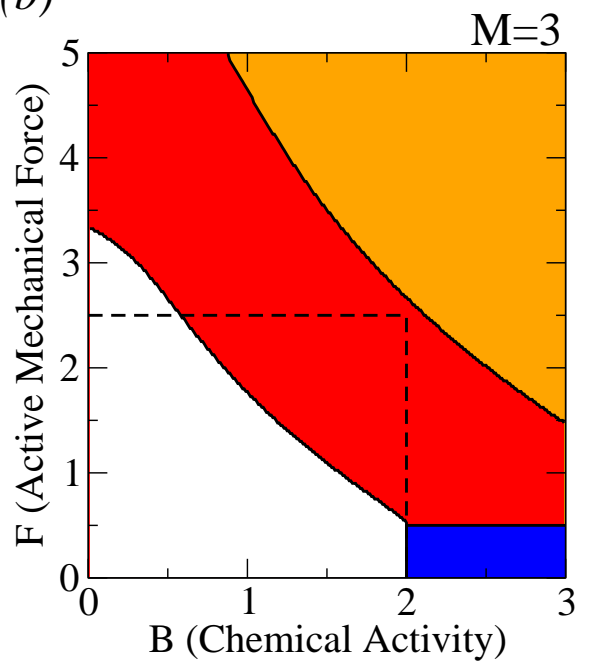

Figure 1: Two analytic phase diagrams in the parameter space spanned by $F$ and $B$, obtained from the dispersion relations for two different values of $M$ : (a) $M=1$ and (b) $M=3$. Three main regions are observed: homogeneous steady state (white), global oscillations (blue), and waves (red and orange). The dispersion relations of the waves in the red domain possess a maximum with complex values (see Fig.3(a)), whereas the maximum of dispersion relation of the wave in the orange domain has purely real values (see Fig. 3(b)). For comparison, the vertical dashed line marks the oscillatory instability of the "pure" Brusselator, whereas the horizontal dashed line indicates the mechanochemical instability in the absence of reaction found in [3]. Crosses show the parameter values used in the dispersion relations plotted in Fig. 3. Parameters of the model: $\ell=1, \rho_{f}=0.5, D_{c}=1, \psi=0.125, A=1$.

corresponds to the ratio of the diffusive to advective time scales. In the absence of any reaction $\psi=0$ and of any elastic components $(E=0)$, the critical Péclet number $(1+A)^{2} / A$ determines the onset of the mechanochemical instability in the absence of reaction [3] and depends on the steady state concentration $c_{o}=A$. The condition $F>1(F<1)$ decides if the homogeneous state is unstable (stable).

\subsection{Phase diagram}

Figure 1 shows the phase diagram of the model, obtained by calculation of the dispersion relations solving Eq. (25). The non-dimensional mechanical 
parameters $F$ and $M$, and the chemical parameter $B$ of the Brusselator are varied to characterize the parameter space. For small values of $B$ and $F$ the homogeneous state is stable. Increasing $B$ and $F$ the homogeneous state becomes unstable through an oscillatory Turing instability and wave solutions are expected. Two types of waves are observed (orange and red domains of Fig. 1). Their characteristics are described in detail in the following section. Furthermore, a region where the Hopf instability leads to global oscillations is also obtained for small values of $F$.

When keeping $F=0$ and increasing the value of $B$ above the critical value $B_{c}$ the homogeneous state becomes unstable through a Hopf bifurcation, that is characteristic for the Brusselator model [54]. On the other hand, in the absence of any reaction, i.e., $\psi=0$, and increasing the value of $F$ above the critical value $F_{c}$, the homogeneous state becomes unstable through a wave instability due to the mechano-chemical properties of the poroelastic medium [3]. When both forces are combined, the homogeneous state becomes unstable for values of $B$ and $F$ below the critical values $B_{c}$ and $F_{c}$, respectively, i.e., in the area below the dashed line in Fig. 1.

The effect of $F$ is partially precluded by an increase of $M$, compare both panels in Fig. 1, as the effect of increasing $M$ shifts the bifurcations to higher values of $F$. A more detailed study of the effect of $M$ is shown in Fig. 2. For $B<B_{c}$ (Fig. 2(a,b)), an increase of $M$ shifts the transition from the stable uniform state to waves towards higher values of $F$. For the case $B>B_{c}$, the behavior is different (Fig. 2(c,d)): The large value of $B$ implies that the system is already unstable due to a Hopf bifurcation for small active forces. Waves appear for $F>0.5$, independently of the value of $M$.

\subsection{Dispersion relations}

Figure 3 shows four examples of characteristic dispersion relations for the behaviors displayed in Fig 1. Their locations on the corresponding phase diagram are indicated in Fig 1(a). For small values of $F$ and $B$ the homogeneous state is stable, see Fig 3(c). Upon increase in the parameter $B$ the characteristic Hopf 

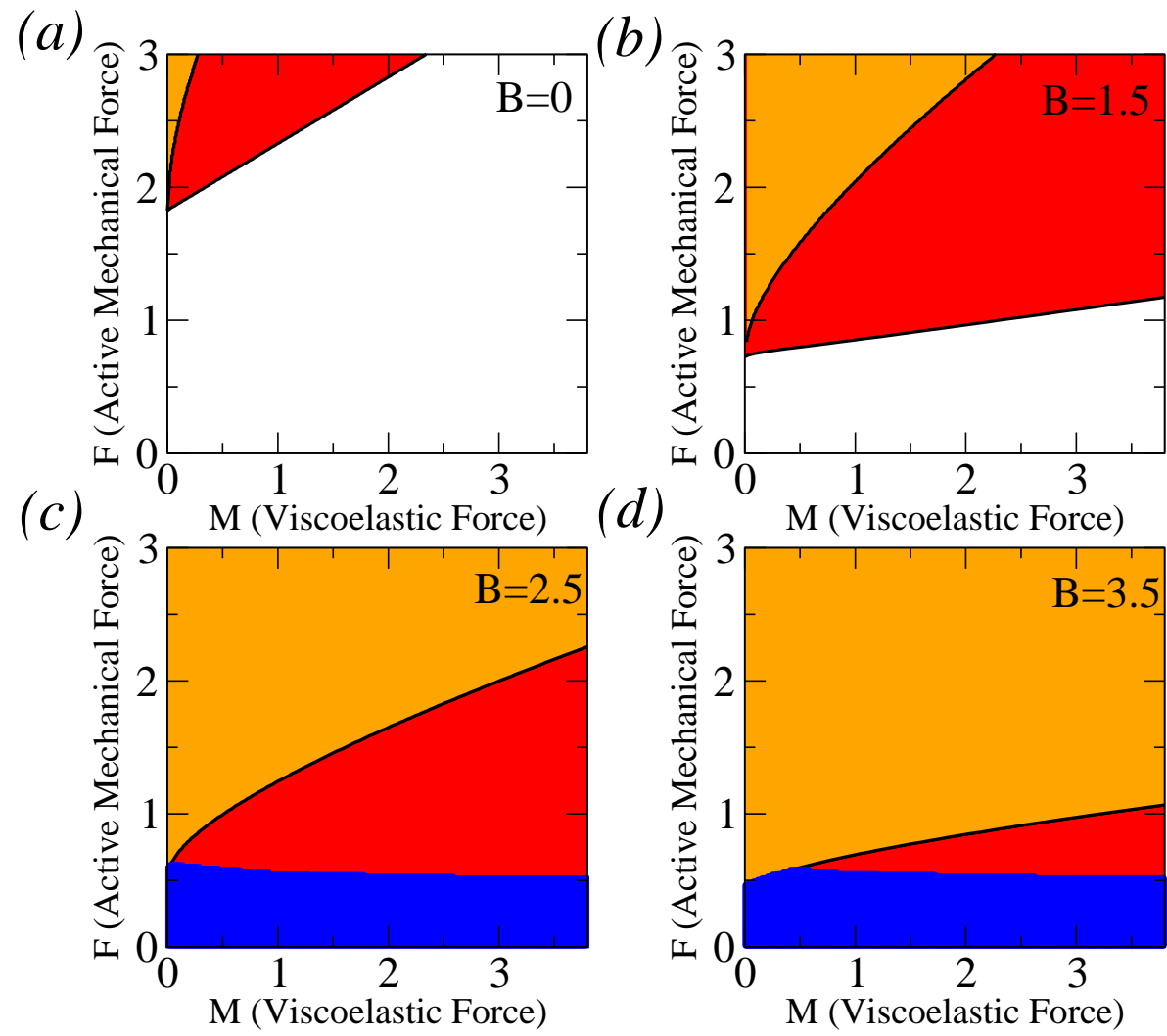

Figure 2: Four analytic phase diagrams in the parameter space spanned by $F$ and $M$, obtained from the dispersion relations for four different values of $B$ : (a) $B=0$, (b) $B=1.5$, (c) $B=2.5$, and (d) $B=3.5$ (b). Three main regions are observed: homogeneous steady state (white), global oscillations (blue), and waves (red and orange). Parameters of the model as in Fig. 1. 

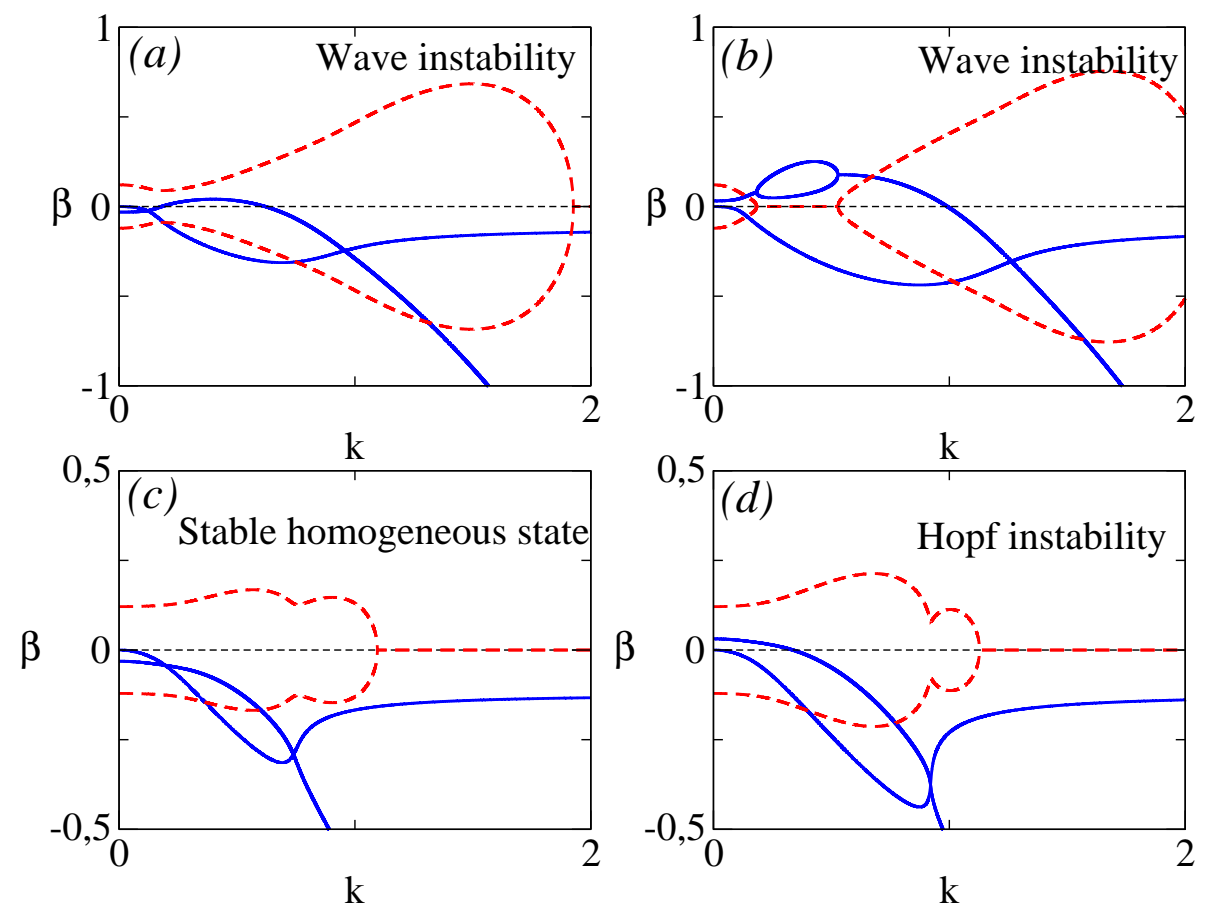

Figure 3: Dispersion relation of the homogeneous solutions for four different values of the parameters of the model: (a) $F=1.5$ and $B=1.5$, (b) $F=2$ and $B=2.5$, (c) $F=0.25$ and $B=1.8$, and (d) $F=0.25$ and $B=2.5$; corresponding to crosses in Fig 1. The thick solid and dashed lines correspond to real and imaginary part of the dispersion relation, respectively. The thin dotted line marks $\beta=0$. Parameters of the model as in Fig. 1.

bifurcation occurs and the maximal eigenvalue is found for $k=0$ (Fig 3(d)). For larger values of $F$, wave instabilities are observed, see Fig 3(a). Further increases of $F$ cause the maximum of the real eigenvalue to become purely real in the interval $0.2 \leq k \leq 0.5$ (Fig. 3(b)), there are, however, unstable eigenvalues with an imaginary component once $k>0.5$, see Fig $3(\mathrm{~b})$.

We distinguish two types of wave instabilities if the largest unstable eigenvalue is either complex or real. For $\psi=0$ the first type of dispersion curve has been found to produce chaotic patterns (Fig 3(a)), while the second type of dispersion curve (see Fig 3(b)) gave rise to travelling domains [3]. Note that with reaction such dynamics may change, because for $\psi>0$ the properties of 
the patterns may depend on the particular value of $\psi$.

\section{Experimental observation of cell thickness oscillations in proto- plasmic droplets of Physarum}

\subsection{Experimental materials and methods}

Plasmodia of the slime mould Physarum polycephalum, strain HU195 $\times$ HU200, were cultured on moist filter paper by feeding with oat flakes (Kölln Flocken) at $21{ }^{\circ} \mathrm{C}$ in the dark. Sclerotia were obtained by drying the wet filter paper on which a sufficient amount of the plasmodium had crawled. Sclerotia were stored in the dark for 3-10 months. The sclerotium is a dormant, dehydrated state of Physarum that may convert back into the plasmodium whence the environmental conditions turn favorable again.

Plasmodia were grown from germinated sclerotia in $9 \mathrm{~cm}$ polystyrene Petri dishes on $1.0 \% \mathrm{w} / \mathrm{v}$ (weight/volume) plain agar gels (Kobe Agar). Protoplasmic droplets were obtained by puncturing a strand (of $1.0-1.5 \mathrm{~mm}$ diameter) of the plasmodial network with a scalpel, collecting $\approx 0.5 \mu \mathrm{l}$ of protoplasm with a micropipette, and depositing the extracted protoplasm on a polypropylene Petri dish containing a nutrient-free, $1.0 \% \mathrm{w} / \mathrm{v}$ plain agar. Within a few seconds the protoplasmic droplet forms a plasma membrane, thus becoming a new cell, which begins its cellular reorganization.

The Petri dish with the protoplasmic droplet is placed under a microscope, covered, and the temperature kept constant at $21.0 \pm 0.5^{\circ} \mathrm{C}$ by the laboratory air conditioning system. The Petri dish was illuminated homogeneously from below by a an array of 60 green LEDs (LedGalaxy, JDR-2760-G, Hamburg, Germany) emitting at $\lambda=517 \pm 20 \mathrm{~nm}$. A sheet of frosted glass and a neutral filter $(1.0$ o.d.) were placed in between the light source and the Petri dish. The dynamics of the protoplasmic droplet was monitored from above through a microscope (Zeiss Stemi SV11, Göttingen, Germany, magnification 19.6×) by an 8 bit charge controlled device (CCD) camera (Hamamatsu Photonics ST70CE, Hamamatsu, Japan). The images were collected at intervals of $4.2 \pm 0.2 \mathrm{~s}$ with 

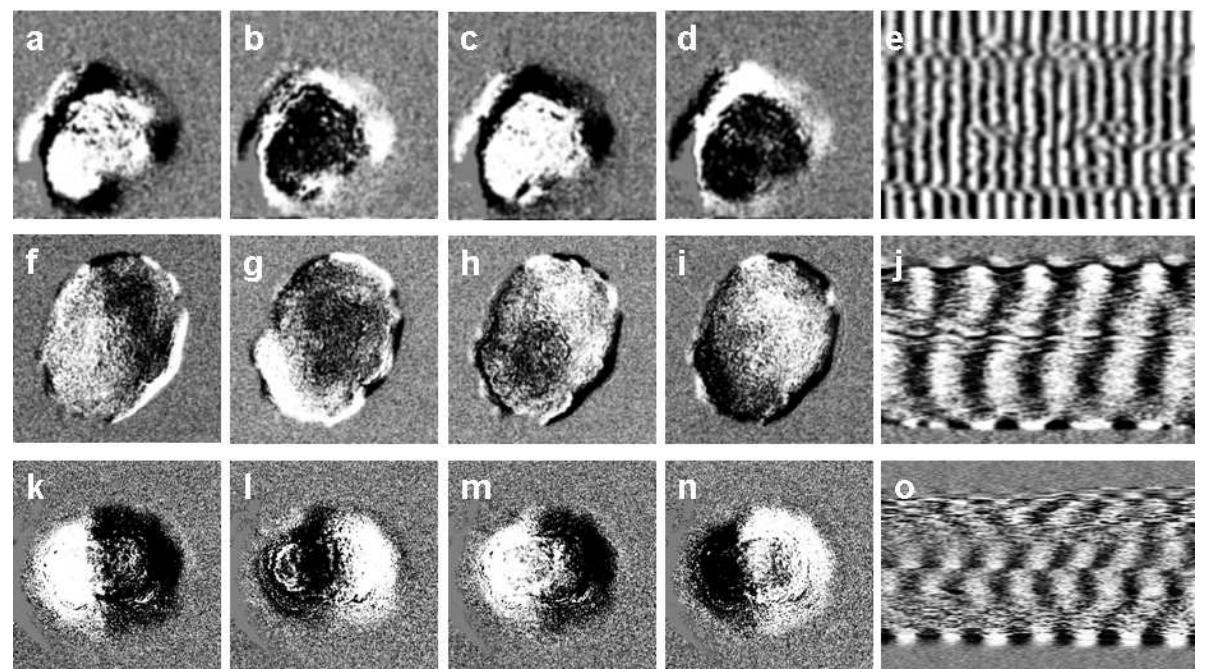

Figure 4: Spatio-temporal patterns of cell thickness oscillations observed in protoplasmic droplets of Physarum polycephalum. Top row: (a-d) snapshots of global oscillations of a global thickness oscillation where the rim oscillates anti-phase to the bulk of the droplet. (e) Time-space plot of global oscillations. Bright and dark pixels indicate a decreasing and increasing cell thickness, respectively. Central row: (f-i) Propagating wave of cell thickness. The wave starts at the top right of the cell and propagates to the down left. (j) Time-space plot of the propagating wave of cell thickness. Bottom row: (k-n) Standing-wave-like pattern of cell thickness, and (o) the corresponding time space-plot.

an image resolution of $768 \times 576 \mathrm{px}$ corresponding to an area of $3.5 \times 2.6 \mathrm{~cm}^{2}$, i.e., a resolution of $0.0456 \mathrm{~mm} / \mathrm{px}$. The frames were transferred to a computer for data processing and analysis.

As the brightness of the transmitted light through the protoplasmic droplets is inversely proportional to their thickness, relative thickness oscillations can be assessed by image analysis. The background was subtracted from all original images. Next, each background-subtracted image was subtracted from its preceding image, and the increase or decrease in thickness was coded by grey levels. Regions, where the thickness of the protoplasmic droplet increased and decreased were coded in bright and dark grey, respectively. 


\subsection{Experimental results}

A droplet of freshly extracted protoplasm forms a cell membrane within a few seconds, thus forming a new cell that contains many nuclei. This freshly formed cell must be considered as being highly disturbed as it was formed from recently extracted protoplasm. This cell begins to reorganize itself and to undergo intracellular differentiation processes in order to form a viable cell.

After the formation of the cell membrane, the protoplasmic droplets remained quiescent for $10-12 \mathrm{~min}$. Then, they began to develop macroscopic oscillations of their cell thickness. These oscillations generally start at the rim of the droplet, forming circular waves of cell thickness at the rim. A few minutes later, the bulk of the cell also started to vary its thickness. Initially these oscillations are aperiodic and irregular in time and space, but after some while, more coherent dynamic structures are formed. In addition to thickness oscillations in the bulk of the droplet, the shape of the droplet oscillates displaying a series of oscillation modes.

Once the bulk oscillations become more coherent, these oscillations can be classified according to their spatio-temporal appearance. For instance, typical oscillatory cell thickness patterns observed are global oscillations (where the rim oscillates in anti-phase with the bulk of the cell; Fig. 4(a-e)), propagating waves of cell thickness (Fig. 4(f-j)), or standing wave-like oscillations (Fig. 4(k-o)). In addition to these patterns of uni-axial symmetry, patterns with different symmetries, such as spiral-shaped waves or pairs of counter-rotating spiral-shaped waves of cell thickness are also observed, as well as aperiodic, irregular cell thickness oscillations. Although these different types of oscillatory patterns may last for some time, all of them transient, and they may easily convert into each other. In our experiments, we did not observe any determined temporal sequel of patterns of thickness oscillations. Rather, the temporal sequel of cell thickness oscillations was random, as was the persistence of the different types of oscillations.

Although the transitions between the different types of thickness oscillations were random, we could observe a certain correlation between the size of the pro- 
toplasmic droplet and the wave form developed. Whereas global cell thickness oscillations as well as standing and travelling-wave type thickness oscillations were more frequently observed in small protoplasmic droplets, counter-rotating spiral-shaped thickness oscillations were more frequently seen in larger protoplasmic droplets, i.e., droplets of diameters larger than $150 \mu \mathrm{m}$. This suggests that the type of thickness oscillations patterns that develop in microplasmodia, may depend on the extension and geometry of the protoplasmic droplet, and hence on the ability of the droplet to accommodate the different forms of oscillations.

\section{Active poroelastic model for Physarum protoplasmic droplets}

In this section we apply the generic poroelastic model described in Section 2 to protoplasmic droplets of Physarum. First, the parameter values of the model are chosen form the literature for the particular case of Physarum. Where physical parameter values for Physarum were not available in the literature, we use values obtained for other types of eukaryotic cells. The chemical parameters $A$, $B$ and $\psi$ are chosen to reproduce the oscillation period of calcium oscillations in Physarum [39, 52]. The stability analysis of the equations is performed using the set of values tabulated in the right column of Table 1. Second, numerical simulations of the model with the chosen parameter values are done and compared with the experimental results.

\subsection{Parameter values}

The cytosol is fluid. Assuming that the cytosol is a diluted aqueous suspension, its viscosity may be assumed to be similar to that of water $\left(\eta_{\text {water }}=10^{-3}\right.$ $P a s$ ). However, it is known that small particles in the cytosol increase this viscosity, and the value of $\eta_{f}$ may increase by a factor two or three [12].

The viscoelastic properties of the cytoskeleton have been extensively measured by atomic force microscopy. Typical values of the elastic (Young) modulus of mammalian cells are between 1 and $10 \mathrm{kPa}$ [55], although cardiac cells may 
Table 1: Parameter values of the model

$\begin{array}{lllll}\text { Symbol } & \text { Meaning } & \text { Units } & \text { Literaturevalues } & \text { Physarum } \\ E & \text { Young modulus } & k P a & 1[12] 1-10[55], 9[57], 50[35] & 9 \\ \eta_{f} & \text { fluid viscosity } & k P a s & 2-3 \cdot 10^{-6}[12,61], 10^{-3}[59] & 2 \cdot 10^{-6} \\ \eta_{g} & \text { gel viscosity } & k P a s & 0.001[45], 0.12[61,58], 300[35] & 0.13 \\ \rho_{f} & \text { solvent fraction } & - & 0.35[11,60], 0.75[12,45] & 0.75 \\ \rho_{g} & \text { gel fraction } & - & 0.25[12,45] & 0.25 \\ \xi & \text { active tension } & k P a & 1.8[61], 2-200[45], 400[35] & 1-10 \\ \ell_{m} & \text { pore size } & \mu m & 0.015[12,60], 0.05[61] 0.2[45] & 0.1 \\ D_{c} & \text { calcium diffusion } & \mu m^{2} / s & 200[67], 370[61], 500[45] & 200 \\ A & \text { Parameter } & - & - & - \\ B & \text { Parameter } & - & - & \end{array}$

have Young moduli larger than $100 \mathrm{kPa}[55,56]$. Experiments with Physarum yielded a Young modulus of $\approx 9 \mathrm{kPa}[57]$.

The viscosity $\eta_{g}$ of the cytoskeleton can be assessed from experiments run with various cell types, for example, for the cytoskeleton in neutrophils a value of $\eta_{g}=130 \mathrm{~Pa} s$ is observed [58]. However, from experiments inducing large deformations in Physarum cells much smaller values are obtained (i.e., $1 \mathrm{Pas}$ [59]).

The volume fractions of the solvent and gel phases are assumed to be constant. By hyperosmotic shocks the minimal fluid fraction of HeLa cells was estimated to be $\rho_{f}=0.35$ from the total decrease of the cell volume $[11,60]$. Recent studies of passive poroelasticity have used a value of $\rho_{f}=0.75$ [12] and, therefore $\rho_{g}=0.25$ for the gel phase. These values, together with the cytosol viscosity yield a renormalized viscosity $\eta=0.09 \mathrm{kPa} s$.

The characteristic mesh size of the porous structure of the cytoskeleton has been calculated from experiments in living cells. Typical results obtained for HeLa cells point to $\ell_{m}=15 \mathrm{~nm}[12,60]$. However, larger values for the porous 


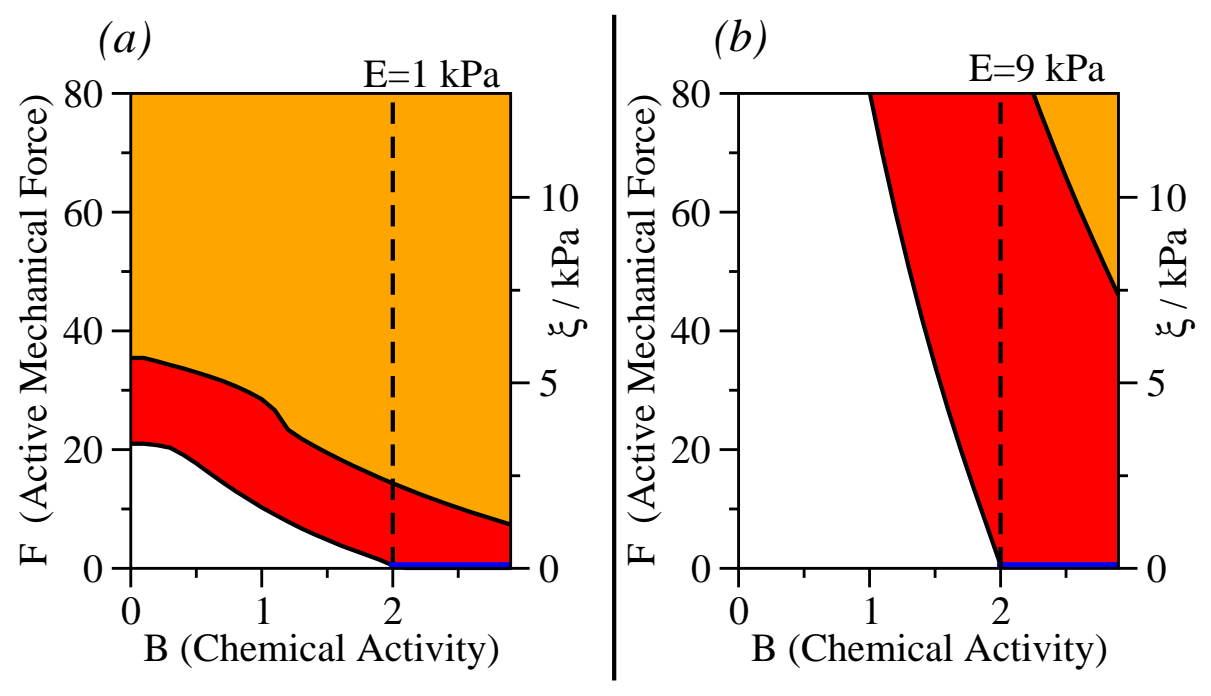

Figure 5: Phase diagram in the parameter space spanned by the active mechanical force $F$, and the parameter $B$ of the Brusselator model as obtained from the dispersion relations for (a) $E=1 \mathrm{kPa}$ and (b) $E=9 \mathrm{kPa}$, using parameter values fitted to Physarum dynamics (Table 1, right column). Three main regions are observed: homogeneous steady state (white), global oscillations (blue), and two domains of wave patterns (red and orange). The dashed line at $B_{c}=2$ indicates the onset of the Hopf instability in the Brusselator model.

structure were used previously in the mathematical modelling of the Physarum cell: $\ell_{m}=50-200 \mathrm{~nm}[45,61]$. Here we have chosen an intermediate value of $\ell_{m}=0.1 \mu \mathrm{m}$. Using this value for the pore size, the permeability $\kappa$ can be obtained from the relation $\kappa=\ell_{m}^{2}$, yielding a value of $\kappa=0.01 \mu m^{2}$. The parameter $\beta$ can be calculated from the solvent viscosity as $\beta=\eta_{f} / \kappa=0.0002$ $k P a s / \mu m^{2}$. Finally, the characteristic length $\ell$ is set to be $\ell=\ell_{m} \sqrt{\eta / \eta_{f}}=21$ $\mu m$.

The concentrations $c$ and $a$ are dimensionless. The steady state of the Brusselator model corresponds to the equilibrium concentration $c=A$ and in order to have comparable variables to the previous studies on active poroelastic media [3], we choose $A=1$. In the limit $\psi=0$ we recover the results shown in ref. [3]. The parameter $\psi$ corresponds to a characteristic time, which is fitted to $\psi=0.125 s^{-1}$ to reproduce the characteristic time scale of the calcium oscilla- 
tions in Physarum with the Brusselator model. The characteristic time scale of the calcium oscillations is found to be $\approx 1 \min [39,52]$. The critical frequency of the limit cycle at the bifurcation is $\omega=\psi A$ [54] which produces a period of $T=2 \pi /(\psi A)$.

\subsection{Phase diagram}

The phase diagram in the phase plane spanned by the mechanical force parameter $F$, which represents the active force, and the parameter $B$ of the Brusselator (i.e., the chemical reaction system) was calculated using the estimated parameter values for Physarum (see Table 1). The phase diagrams were calculated using two different values for the elastic (Young) modulus E (Fig. 5). Similarly to the parameter space of the generic model shown in Fig. 1, a homogeneous steady state and wave patterns dominate the phase diagram (Fig. 5). The domain of global oscillations is restricted to a smaller area of the phase diagram in comparison with the generic phase diagram (Fig. 5), because we obtain large values of the active mechanical force $F$ using the parameters estimated for Physarum.

For smaller values of $\mathrm{B}\left(\mathrm{B}<\mathrm{B}_{c}=2\right)$, the increase of the active mechanical force $F$ destabilizes the homogeneous steady state through the appearance of a wave instability. For larger values of the chemical parameter $\left(B>B_{c}\right)$, the active mechanical force $F$ causes a change from global oscillations to a wave instability. Further increase of the active force $F$ alters the structure of the dispersion relation and more complex patterns may arise [3].

The change of the value of the Young modulus $E$ between both panels in Fig. 5 leads to two different values of $F$ that are needed for the instability to occur. In the absence of any reaction, i.e., $\psi=0$, the critical values of the control parameter are $F=38.5$ and $F=338.5$, for $E=1 k P a$ and $E=9 k P a$, respectively.

\subsection{Numerical results}

We integrate Eqs. (19-22) numerically in a one-dimensional domain using a finite differences method. We assume a fixed droplet without motion of the 
membrane boundaries, therefore, we impose zero-flux boundary conditions for $c$ and $a$ and Dirichlet conditions for the fields $w$ and $u$, i.e., $w(0)=w(L)=0$ and $u(0)=u(L)=0$.

The process of pattern formation depends on the initial condition. We start with a homogeneous spatial distribution of the biochemical concentrations $a, c$ of the control and regulator species, respectively. This initial distribution is perturbed to yield the formation of spatio-temporal patterns. Here, small random perturbations are added to the initial homogeneous state to generate waves, whereas the application of large homogeneous perturbations to the steady state lead to the emergence of more coherent wave patterns, closer to global oscillations.

Figure 6 shows the spatio-temporal dynamics of the concentration $c$ and the thickness $h$ for different parameters. We estimate the relative change in thickness $\delta h=\left(h-h_{0}\right) / h_{0}$ with respect to the reference configuration $h_{0}$ to be proportional to $\partial_{x} u$. This approximation is justified by the experimental observation that regions in Physarum microplasmodia deform isotropically [34]. If $z$ represents the coordinate orthogonal to $x$, the height is approximated by $\delta h \approx \partial_{z} u_{z}$. Then, isotropy gives $\delta h \approx \partial_{z} u_{z} \propto \partial_{x} u_{x}=\partial_{x} u$. This means that large (small) values of $\partial_{x} u$ imply a local increase (decrease) of thickness of the cell.

Travelling mechano-chemical waves are observed in the numerical simulations under different conditions. Different examples are displayed in Fig. 6. The boundary conditions produce inhomogeneous velocities of the waves and large increases of both the concentration $c$ and the thickness $h$ at one of the extremes of the system. Such inhomogeneity in the deformation may produce the motion of the whole droplet with free boundary conditions. The position where the waves set in differs depending on the realization. In particular, Fig. 6(e) shows a pacemaker generated close to the center of the system. More complex dynamics is shown in Fig. 6(f), where travelling waves are generated alternatively in both directions. This alternating dynamics in one dimension resembles the spiral wave dynamics in two dimensions. 


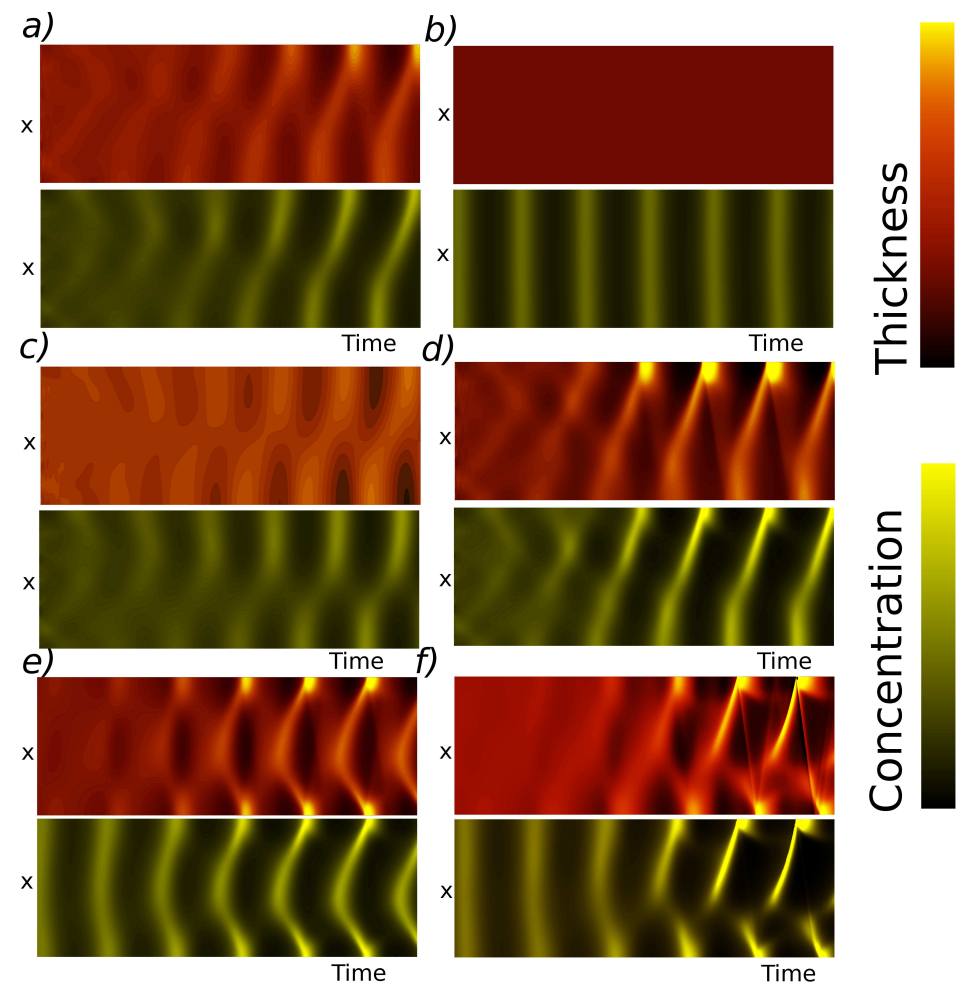

Figure 6: Spatio-temporal evolution of the concentration $c$ and the local thickness $h$ for four different values of the parameters: $\xi=1 k P a(F=6.25)$, and $E=5 k P a$ (a) and (b); $\xi=1$ $k P a(F=6.25)$, and $E=9 k P a(c) ; \xi=2 k P a(F=12.5)$, and $E=5 k P a(d)$ and (e); and $\xi=5 \mathrm{kPa}(F=31.25)$, and $E=9 \mathrm{kPa}$ (f) Parameter values of the Brusselator model $A=1$ and $B=2.1$. Two types of initial conditions are employed: random (a) (c) and (d), and slightly noisy homogeneous oscillation (b) and (e). Numerical parameter values: $\Delta x=2$ $\mu m, L=10^{3} \mu m, \Delta t=10^{-5} \mathrm{~s}$ and $T=500 \mathrm{~s}$. 
Wave structures with velocities of $5-30 \mu \mathrm{m} / \mathrm{s}$ have been observed in Physarum [35]. In our experiments, we observe travelling waves of cell thickness (Fig. 4(fj)), the velocities of which are $\approx 15 \mu \mathrm{m} / \mathrm{s}$. Such values are close to the wave velocities observed in the numerical simulations, which are $\approx 25 \mu \mathrm{m} / \mathrm{s}$.

Small amplitude standing waves are also shown in Fig. 6(c). They appear at small values of the active tension and they seem to be transitory. Standing waves have been also observed in the experiments (Fig. 4(k-o)).

Finally, global oscillations have also been observed in experiments where the bulk of the cell oscillates in anti-phase to its rim (Fig. 4(a-e)). The periodically oscillating dynamics of the bulk of the cell can be reproduced by numerical simulations using the appropriate initial conditions, see Fig. 6(b). The mechanical deformation depends on the gradient of the active force, therefore, the mechanical part remains static, and no deformation results. Under the same parameter values, but with different initial conditions, travelling waves appear, see Fig. 6(a). It may indicate that such global oscillations are unstable as in Figs 6(e,f).

The condition of small strain $\partial_{x} u \ll 1$ used in the model derivation constrains the parameter space, where our model is applicable. The value of $\partial_{x} u$ increases with active mechanical force $F$, thus, large values of the active force were not employed in our numerical simulations. The strain also depends on the elastic modulus $E$ as small values of the modulus lead to large deformations and hence violate the condition of small strain. In such limits, turbulent and complex dynamics are expected, already in the absence of any biochemical reaction [3].

\subsection{Spatio-temporal properties of the waves}

The dispersion relations employed for the calculation of the phase diagram shown in Fig. 5 are also used for the study of the spatio-temporal properties of the non-linear patterns observed in the numerical simulations (Fig. 6).

First, we consider the characteristic length obtained from the dispersion relation. The most unstable mode is typically correlated to the wavelength $\lambda$ 

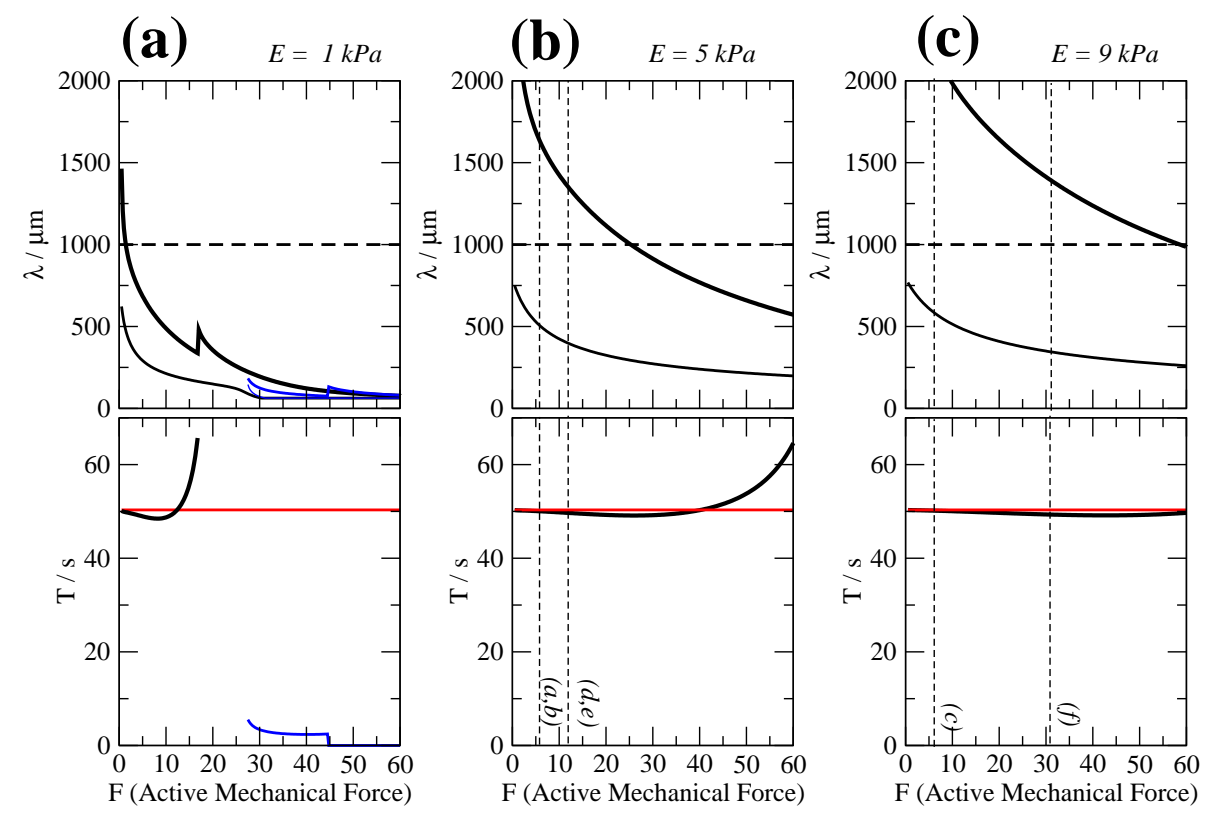

Figure 7: Characteristic wavelength and period obtained from the linear stability analysis of Eqs. (19-22) for the parameters chosen to model Physarum for different values of the elastic modulus: $E=1 k P a(a), E=5 k P a(b)$ and $E=9 k P a(c)$ and $B=2.1$. For each value the characteristic wavelength (top panels) and period (bottom panels) are shown. In top panels the wavelengths associated to the most unstable mode (thick line) and the smallest wavelengths of an unstable mode (thin line) are shown in comparison with the size of protoplasmic droplets of Physarum (dashed line) employed in the simulations. In the bottom panels the periods associated to the most unstable mode are shown (thick black line) when it exists and compared with the results of the Brusselator model with the same parameters (red line). For the two panels in (a) the case $\mathrm{B}=0$ is also shown for comparison (blue lines). Vertical thin dashed lines correspond to the values employed in the numerical simulations shown in Fig. 6 . 
of the patterns obtained in the full numerical simulations (Fig. 3). In Fig. 7 we show the dependence of the wavelength $\lambda$ on the parameter $F$. If $F \rightarrow 0$ the maximum corresponds to $k=0$ because we recover the Hopf instability (we employ $B>B_{c}$ ). For larger values of $F$ the characteristic wavelength shrinks to more realistic values in comparison with the size of the droplets $(1 \mathrm{~mm})$. Pattern formation is, however, possible for small values of $F$ because there are unstable modes at smaller wavelength than the size of the droplets, for instance, the wavelength associated to the larger unstable mode of the dispersion relation (Fig. 7). Such unstable modes explain the patterns observed in the numerical simulations as shown in Fig. 6. In fact, the thin vertical dashed lines in Fig. 7 correspond to parameter values of the numerical results in Fig. 6. For large values of $F$ the wavelengths converge to the values obtained in the system with linear reaction $(B=0)$, see blue lines in Fig. 7(a), and indicate that the viscoelastic properties of the model determines the final wavelength in the active poroelastic model.

On the other hand, the results from the temporal dynamics based on the dispersion relation are different. For some conditions the imaginary part of the eigenvalues gives rise to oscillatory periods close to the period obtained directly from the reaction model, without any type of transport, as seen from the comparison of the black and red lines in the bottom panels of Fig. 7. The oscillation frequency is almost constant in Fig. 7(b-c), and it explains that the characteristic periods of the waves in Fig. 6 are independent of the parameters $F$ and $E$.

\section{Discussion}

We have combined an active poroelastic model for the cellular cytoplasm with a generic reaction-diffusion model of a biochemical oscillator. Linear stability analysis and numerical simulations show the appearance of travelling and standing waves. The resulting model, supplied with realistic parameter values from the experimental literature, reproduces the patterns obtained in our 
experiments with protoplasmic droplets of Physarum polycephalum.

Our experiments on protoplasmic droplets show a large variety of behaviors and different patterns of contraction of the cell thickness are found. In contrast to a previous report [31], we do not observe any temporal directionality among the appearance of patterns of cell thickness oscillations. The different patterns appear randomly without any particular order. The protoplasmic droplet is in the midst of a reorganization process to form a microplasmodium and, therefore, the different parameters values determining viscoelastic and active properties are continuously evolving with time. This parameter evolution highly depends on the particular microplasmodia. For this reason, the comparison of the phenomenological observations with the model results are of crucial importance, because the numerical simulations also show a rich variety of dynamic cell thickness patterns as the parameter values are slightly varied.

A single active poroelastic system without any biochemical oscillator produces waves and complex dynamics [3]. In the particular case of Physarum the mechanical deformations are coupled with calcium oscillations [32]. In the present paper, we have shown that the combination of both effects, namely poroelasticity and a biochemical oscillator enhances the region of pattern formation in comparison to a model that neglects the contribution of the biochemical oscillator. Furthermore, we have shown that whereas the period of oscillations is strongly controlled by the biochemical model employed, the spatial properties of the patterns are determined by the viscoelastic properties of the system.

The typical wavelength of the patterns obtained using the parameter values for Physarum (Table 1) range from $250-1000 \mu m$ (Fig. 7(c)). It is noteworthy that these wavelengths correspond quite well to the wavelengths observed experimentally in protoplasmic droplets but also to the wavelengths of undulations formed by a finger instability at the leading edge (the so-called apical zone) of extended macroplasmodia in Physarum [65]. Similarly to the protoplasmic droplets the apical zone of extended plasmodia consists of a porous medium.

The active poroelastic system is defined by the velocity of the fluid (i.e., the cytosol) and the deformation of the gel (that is, the cytoskeleton). Similar 
descriptions in terms of active materials have been used to model the cytoplasm [10] as well as to describe cortical flows in polarized cells [66] as well as aspects of cell motility [9]. The cytoplasm has also been modelled as an active fluid that can transport the molecular motors [2] which at the same time produce active tension in the fluid [2]. In contrast, here we considered the poroelasticity of the cytoskeleton [12].

Models assuming an active cytoplasm [2, 62] provide an alternative approach for the formation of patterns in the interior of cells compared to the standard reaction-diffusion systems, which built an combination of diffusive transport of molecules in the cell with biochemical reactions between different species. Such models have been employed for example to explain cell polarity [63] or cellular division of E. coli [64].

The latter examples explicitly consider different reactions inside the cell. In the present paper, we have considered a simple qualitative reaction model addressing the calcium oscillator in Physarum. However, the main details of the calcium oscillator can be analyzed and a more complicated model can be derived [45]. It may permit more quantitative comparison with experimental results. However, the detailed mechanism coupling the calcium oscillations and the mechanical contractions is still an open issue $[35,39,42,43]$ that calls for further experimental studies.

A more complex description of the viscoelastic properties may also improve the comparison with experiments. We have employed the Kelvin-Voigt model for viscoelasticity which is a good model for short times. However, for some applications with slow processes, one may consider the use of a different elastic model like the Maxwell model of viscoelastic fluids. Moreover, we have restricted our calculations to linear viscoelasticity which is not true for large deformations of the cytoplasm, where nonlinear elasticity need to be included.

We have considered here a simple one-dimensional system to analyze the equations and to perform the numerical simulations. Obviously, two- or even three-dimensional systems provide more complete descriptions. In the present paper, for the sake of simplicity, we restrict our study to the one-dimensional 
case which, nevertheless, allows the investigation of uniaxial patterns and their comparison to corresponding experiments.

In summary, we have extended the one-dimensional active poroelastic model derived in [3] by introduction of a biochemical oscillator. For simplicity, we have employed the generic Brusselator oscillator. The resulting model reproduces a variety of experimentally observed uniaxial patterns observed in protoplasmic droplets of Physarum polycephalum.

\section{Acknowledgements}

We acknowledge financial support from the German Science Foundation DFG within the frameworks of SFB 910 "Control of self-organizing nonlinear systems" and GRK 1558 "Nonequilibrium Collective Dynamics in Condensed Matter and Biological Systems". SA acknowledges support by MINECO of Spain under the Ramon y Cajal program with the grant number RYC-201211265. 
Appendix 
Table 2: List of variables

\begin{tabular}{|c|c|c|}
\hline Symbol & Definition & Meaning \\
\hline$a$ & & control species \\
\hline$a$ & & concentration of the control species \\
\hline$c$ & & regulator species (calcium ions) \\
\hline$c$ & & concentration of the regulator species (calcium ions) \\
\hline$D_{a}$ & & diffusion coefficient of the control species \\
\hline$D_{c}$ & & diffusion coefficient of the regulator species \\
\hline$E$ & & Young modulus \\
\hline$F$ & $=\frac{\xi A}{D_{c} \beta(1+A)^{2}}$ & non-dimensional mechanical parameter, active mechanical force \\
\hline$f_{f}$ & & drag force in the cytosol \\
\hline$f_{g}$ & & drag force in the cytoskeleton \\
\hline$h$ & & local cell thickness \\
\hline$h_{0}$ & & average cell thickness \\
\hline$\ell$ & $=\sqrt{\eta / \beta}$ & characteristic length \\
\hline$\ell_{m}$ & & cytoskeletal mesh size \\
\hline$M$ & $=\frac{E}{D_{c} \beta}$ & non-dimensional elastic parameter \\
\hline$p$ & & pressure \\
\hline$R_{a}(c, a)$ & & reaction kinetics of the control species \\
\hline$R_{c}(c, a)$ & & reaction kinetics of the regulator species \\
\hline$T$ & & active tension \\
\hline$T_{0}$ & & active tension at low $c$ \\
\hline$u$ & & local displacement field \\
\hline$v$ & & velocity fluid filed \\
\hline$w$ & $=v-\dot{u}$ & body reference velocity \\
\hline
\end{tabular}


Table 3: List of variables - continuation

$\begin{array}{lll}\text { Symbol } & \text { Definition } & \text { Meaning } \\ \beta & =\eta_{f} / \kappa & \text { ratio of viscosity of the sol phase to its permeability } \\ \epsilon & \text { strain } \\ \eta & =\rho_{f} \eta_{g}+\rho_{g} \eta_{f} & \text { renormalized viscosity } \\ \eta_{f} & \text { fluid viscosity } \\ \eta_{g} & \text { gel viscosity } \\ \kappa & \text { permeability of the sol phase } \\ \lambda & \text { wavelength of the (wave) patterns } \\ \xi & \text { coupling strength between concentration of regulator and active tension } \\ \rho_{f} & \text { sol fraction } \\ \rho_{g} & \text { gel fraction } \\ \sigma & \text { total stress } \\ \sigma_{f} & \text { stress in sol (cytosolic) phase } \\ \sigma_{g} & \text { stress in gel (cytoplasmic) phase } \\ A & \text { Parameter of the Brusselator model } \\ B & \text { Parameter of the Brusselator model } \\ \psi & \text { Time scale of reaction kinetics } R_{a}(c, a) \text { and } R_{c}(c, a) \\ & \end{array}$




\section{References}

[1] T. Betz, D. Koch, Y.-B. Lu, K. Franze, and J. A. Käs, Proc. Natl. Acad. Sci. USA 108 (2011) 13420-13425.

[2] J. S. Bois, F. Jülicher and S. W. Grill, Phys. Rev. Lett. 106 (2011) 028103.

[3] M. Radszuweit, S. Alonso, H. Engel, and M. Bär, Phys. Rev. Lett. 110 (2013) 138102 .

[4] S. Banerjee and M. C. Marchetti, Soft Matter 7 (2011) 463.

[5] M. H. K. Köpf and L. Pismen, Soft Matter 9 (2013) 3727.

[6] M. H. K. Köpf and L. Pismen, Physica D 259 (2013) 48.

[7] S. Banerjee, K. J. Utuje, and M. C. Marchetti, Phys. Rev. Lett. 114 (2015) 228101.

[8] F. Huber, J. Schnauss, S. Rönicke, P. Rauch, K. Müller, C. Fütterer, and J. Käs, Adv. Phys. 62 (2013), 1-112.

[9] F. Jülicher, K. Kruse, J. Prost, and J. F. Joanny, Phys. Rep. 449 (2007), 3.

[10] J. F. Joanny and J. Prost, HFSP J. 3 (2009), 94-104.

[11] G. T. Charras, M. Coughlin, T. J. Mitchison, and L. Mahadevan, Biophys. J. 94 (2008), 1836-1853.

[12] E. Moeendarbary, L. Valon, M. Fritzsche, A. R. Harris, D. A. Moulding, A. J. Trasher, E. Stride, L. Mahadevan, and G. T. Charras, Nature Materials 12 (2013), 253-261.

[13] K. E. Wohlfarth-Bottermann, Protoplasma 54 (1962) 514-539.

[14] G. Isenberg and K. E. Wohlfarth-Bottermann, Cell Tiss. Res. 173 (1976) 495-582. 
[15] W. Stockem and K. Brix, Int. Rev. Cytol. 149 (1994) 145-215.

[16] N. Kamiya, Protoplasmologia 8 (1959) 1-199.

[17] K. E. Wohlfarth-Bottermann, J. Exp. Biol. 81 (1979) 15-32.

[18] H. Aldrich and J. Daniels (Eds), Cell biology of Physarum and Didynium, vol. 1, Academic Press, New York, 1982.

[19] A. Tero, S. Takagi, S. Saigusa, K. Ito, D. P. Bebber, M. D. Fricker, K. Yumiki, R. Kobayashi, and T. Nakagaki, Science 327 (2010) 439-442.

[20] W. Baumgarten, T. Ueda, and M. J. B. Hauser, Phys. Rev. E 82 (2010) 046113.

[21] M. Ito, R. Okamoto, and A. Takamatsu, J. Phys. Soc. Jpn. 80 (2011) 074801.

[22] A. Fessel, C. Oettmeier, E. Bernitt, N. Gauthier, and H.-G. Döbereiner, Phys. Rev. Lett. 109 (2012) 078103.

[23] W. Baumgarten and M. J. B. Hauser, Phys. Biol. 10 (2013) 026003.

[24] K. Matsumoto, S. Takagi, and T. Nakagaki, Biophys. J. 94 (2008) 24922504 .

[25] R. D. Guy, T. Nakagaki, and W. B. Wright, Phys. Rev. E 84 (2011) 016310.

[26] K.-I. Ueda, S. Takagi, and T. Nakagaki, Phys. Rev. E 83 (2011) 021916.

[27] J.-P. Rieu, H. Delanoë-Ayari, S. Takagi, Y. Tanaka, and T. Nakagaki, J. R. Soc. Interface 12 (2015) 20150099.

[28] O. L. Lewis, S. Zhang, R. D. Guy, and J. C. del Álamo, J. R. Soc. Interface 12 (2015) 20141359.

[29] B. Rodiek, S. Takagi, T. Ueda, and M. J. B. Hauser, Eur. Biophys. J. 44 (2015) 349-358. 
[30] B. Rodiek and M. J. B. Hauser, Eur. Phys. J. Special Topics 224 (2015) 1199-1214.

[31] S. Takagi and T. Ueda, Physica D 237 (2008) 420-427.

[32] S. Yoshiyama, M. Ishigami, A. Nakamura, and K. Kohama, Cell Biol. Int. 34 (2010) 35-40.

[33] S. Tsuda and J. Jones, Biosystems 103 (2011) 331-341.

[34] K. Brix, J. Kukulies, and W. Stockem, Protoplasma 137 (1987) 156-167.

[35] Yu. M. Romanovskii and V. A. Teplov, Phys. Usp. 38 (1995) 521-543.

[36] T. Hasegawa, S. Takahashi, H. Hayashi, and S. Hatano, Biochemistry 19 (1980) 2677-2683.

[37] G. F. Oster and G. M. Odell, Physica D 12 (1984) 333-350.

[38] K. Kohama, Adv. Biophys. 23 (1987) 149-182.

[39] D. A. Smith and R. Saldana, Biophys. J. 61 (1992) 368-380.

[40] H. Satoh, T. Ueda, and Y. Kobatake, Cell Struct. Funct. 9 (1984), 37-44.

[41] H. Satoh, T. Ueda, and Y. Kobatake, Cell Struct. Funct. 7 (1982), 275-283.

[42] V. A. Teplov, Yu. M. Romanovsky, and O. A. Latushkin, Biosystems 24 (1991) 269-289.

[43] V. A. Teplov, Biophysics 55 (2010) 987-995.

[44] M. Radszuweit, H. Engel, and M. Bär, Eur Phys. J. Special Topics 191 (2010) 159-172.

[45] M. Radszuweit, H. Engel, and M. Bär, PLoS One 9 (2014) e99220.

[46] H. Karcher, J. Lammerding, H. Huang, R. T. Lee, R. D. Kamm, and M. R. Kaazempur-Mofrad, Biophys. J. 85 (2003) 3336-3349. 
[47] A. C. Callan-Jones and F. Jülicher, New. J. Phys. 13 (2011) 093027.

[48] W. S. Slaughter, The linearized theory of elasticity, Birkhäuser, Boston, 2002.

[49] W. Alt and M. Dembo, Math. Biosci. 156 (1999) 207-228.

[50] S. S. Rosenfeld, A. Houdusse, and H. L. Sweeney, J. Biol. Chem. 48 (2005) 6072-6079.

[51] K. V. Kumar, J. S. Bois, F. Jülicher, and S. W. Grill, Phys. Rev. Lett. 112 (2014) 208101.

[52] A. C. Durham and E. B. Ridgway, J. Cell. Biol. 69 (1976) 219-223.

[53] I. Prigogine and R. Lefever, J. Chem. Phys. 48 (1968) 1695-1700.

[54] A. De Wit, D. Lima, G. Dewel, and P. Borckmans, Phys. Rev. E 54 (1996) 261.

[55] T. G. Kuznetsova, Micron 38 (2007) 824-833.

[56] A. B. Mathur, A. M. Collinsworth, W. M. Reichert, W. E. Kraus, and G. A. Truskey, J. Biomech. 34 (2001) 1545-1553.

[57] C. H. Norris, J. Cell. Comp. Physiol. 16 (1940) 313-322.

[58] M. A. Tsai, R. S. Frank, and R. E. Waugh, Biophys. J. 66 (1994) 2166-2172.

[59] M. Sato, T. Z. Wong, and R. D. Allen, 1983 J. Cell Biol. 97 (1983) 10891097.

[60] G. T. Charras, T. J. Mitchison, and L. Mahadevan, J. Cell Sci. 122 (2009) 3233.

[61] M. Dembo, Biophys. J. 55 (1989) 1053-1080.

[62] R. Peter, V. Schaller, F. Ziebert, and W. Zimmermann, New. J. Phys. 10 (2008) 035002. 
[63] S. Alonso and M. Bär, Eur. Phys. J. Nonlinear Biomedical Physics 2 (2014) $1-18$.

[64] M. Howard and K. Kruse, J Cell Biol. 168 (2005) 53336.

[65] W. Baumgarten and M. J. B. Hauser, Europhys. Lett. 108 (2014) 50010.

[66] M. Mayer, M. Depken, J. S. Bois, F. Jülicher and S. W. Grill, Nature 467 (2010) 617-621.

[67] S. Rüdiger, Phys. Rep. 534 (2014) 39-87. 